\title{
Using Clinical Pharmacy Support Technicians to Optimize Pharmaceutical Care in the Intensive Care Unit
}

\author{
Vincent H Mabasa, Douglas L Malyuk, Anthony Tung, Robert M Balen, Tricia R Nicolls, \\ and Nicole L Rahiman
}

\section{INTRODUCTION}

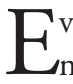
ven as the scope of pharmacy practice expands, impediments to optimal delivery of pharmaceutical care remain. These include shortages of pharmacists, increasing complexity of medication regimens, and increasing acuity of patients and the associated workload. Pharmacy technicians are well positioned to augment direct patient care services because of their knowledge of the medication distribution system in their respective health care centres. At the Royal Columbian Hospital, a 400-bed tertiary health care centre, pharmacy technicians play a large role in the preparation and distribution of medications. This has allowed pharmacists to be deployed primarily to settings where they practise direct patient care. Pharmacy technicians have not, however, been incorporated to any great extent into pharmacists' direct patient care processes and workflows.

The adult tertiary intensive care unit (ICU) at the Royal Columbian Hospital has 26 beds. Patients in the ICU are cared for by 2 multidisciplinary health care teams. Each team is led by an intensivist and includes one clinical pharmacy specialist. Patient care rounds are performed daily from 0915 until about 1300 . The clinical pharmacy specialist typically has $2 \mathrm{~h}$ to do a comprehensive work-up for more than a dozen patients before patient care rounds. During patient care rounds, the health care team reviews the information about each patient sequentially and sets up a plan for treatment for the day. The patients' medications are also reviewed at this time. The clinical pharmacy specialist is expected to be present during patient care rounds and to bring to the team's attention any drug-related problem that has been identified. Because of the increasing demand for ICU beds and the lack of physical space in the current unit, patients requiring mechanical ventilation, who are followed by the ICU health care teams, are sometimes located on units outside the ICU, such as the Emergency Department, the PostAnesthesia Care Unit, or the Cardiac Surgery ICU.

The use of pharmacy technicians to support direct patient care services in the ICU has previously been reported by Ervin and others, ${ }^{1}$ who described the role of the data analyst technician. According to those authors, the activities of data analyst technicians in the ICU focus on retrieving data for individual patients. The information collected may include values for hemodynamic and respiratory parameters, demographic characteristics, fluid and nutritional status, and use of vasopressors. This support activity is valuable to the delivery of pharmaceutical care in the ICU because it allows pharmacists to focus more on cognitive-based activities, which increases their overall work efficiency. However, we felt that pharmacy technicians were capable of performing more value-added activities than had previously been reported and that they could play a larger role in the ICU. We hypothesized that incorporating a clinical pharmacy support technician (CPST) program in the delivery of direct patient care in the adult tertiary-level ICU would further increase pharmacists' work efficiency. We defined work efficiency in terms of the number of patients that the pharmacist could comprehensively assess per day and the time spent on cognitive-based activities. This article describes the CPST program at the Royal Columbian Hospital, the activities performed by the technician in the ICU, the benefits of the program, and considerations for implementing similar programs elsewhere.

\section{DESCRIPTION OF THE PROGRAM}

The CPST program at the Royal Columbian Hospital was established in 2006 to assist clinical pharmacists providing direct patient care services in the general medicine/surgery units and in the ICU. At the time this article was prepared, the job description for a CPST included the following activities: obtaining, organizing, and distributing patient profiles, related material, and support documentation for review by the clinical pharmacy specialist; assisting with the retrieval of drug information; evaluating drug utilization; printing reports; providing discharge counselling; and assisting with various projects. The CPSTs continued to be scheduled for traditional drug-distribution 
rotations in the central dispensary to ensure that they maintained their skills in this area of practice.

The training of CPSTs was supervised by ward-based pharmacists, the clinical pharmacy practice leader, and previously trained technicians involved in the program. Training activities included shadowing ward-based pharmacists, including attending multidisciplinary rounds; guided activities, such as data collection; group discussions; and quality assurance measures. Each trainee received an overview of the variety of information sources available, such as patients' medical records, the computer system, and monitoring forms. In addition, detailed protocols and procedures have been set up to help the CPST perform each activity with consistency, according to expected competencies. Monthly meetings were used to help the technicians to upgrade their skills, as well as for continuing education and quality assurance.

At the time this article was prepared, in mid-2009, 4 CPSTs were involved in the program, providing service from Monday to Friday on a 4-week rotation. The rotation consisted of 1 week of clinical support on the general medicine/surgery floor, 1 week of clinical support in the ICU, and 2 weeks of traditional drug-distribution activities in the dispensary of the central pharmacy.

\section{DUTIES OF THE CPST IN THE ICU}

At the Royal Columbian Hospital, CPSTs have been playing an important role in the delivery of pharmaceutical care in the ICU. They have provided assistance to the clinical pharmacy specialists by assisting with the technical components of the pharmacists' practice. At the time this article was prepared, the CPSTs were performing the activities described below in the ICU, under the supervision of the clinical pharmacy specialists.

\section{Triage of Patients}

To facilitate the delivery of pharmaceutical care by the clinical pharmacy specialists, the CPST helps to keep track of admissions, transfers, and discharges of all critically ill patients in the hospital throughout the day. Each morning, the CPST generates a list of patients who have been admitted to the ICU, and then compares the newly generated list with the previous day's list, notifying the clinical pharmacy specialist of any patient who has moved to a different unit or a different hospital (transfers). This alerts the clinical pharmacy specialist in the ICU to contact the receiving clinical pharmacists to inform them of any pertinent drug-related issues needing follow-up. In addition, if a patient is being transferred out of the unit unexpectedly, which usually occurs because another patient requires the bed, the CPST alerts the clinical pharmacy specialist so that he or she can ensure that the transfer orders are accurate. This activity expedites the movement of patients. At the same time, the clinical pharmacy specialist is informed of any new patients admitted to the ICU, to allow priorities for patient work-up to be adjusted accordingly.
Throughout the day, the CPST keeps track of hospital inpatients who may be referred to the ICU team. The CPST can identify these patients with the help of the clinical pharmacy specialist or by identifying patients who are receiving mechanical ventilation elsewhere in the hospital. Once such a patient is identified, the CPST can proceed with the initial work-up by starting a patient monitoring form and collecting information for predefined parameters. The CPST also notifies the pharmacy dispensary about these patients and resolves any potential drug-distribution problems associated with transferring care to the ICU team. Such problems might include medications going missing during transfer and the need to expedite "stat" medication orders. With the CPST tracking the patients, issues originating in the dispensary or raised by other members of the health care team can be resolved or referred to the pharmacist in an efficient manner. Using up-to-date information about patients' movements, as provided by the CPST, the clinical pharmacy specialist is able to triage his or her priorities accordingly.

\section{Data Collection}

The ICU CPST performs various data-collection duties consistent with those described previously in the literature, ${ }^{1}$ including obtaining, organizing, and distributing patient profiles, related material, and support documentation for the clinical pharmacy specialist. While a patient is under the care of one of the ICU teams, the CPST monitors day-to-day progress by tracking targeted patient-specific parameters and transferring the information to the monitoring form used by the clinical pharmacy specialist (see Online Appendix 1, at www.cjhponline.ca/index.php/cjhp/issue/view/72/showToc). This allows the clinical pharmacy specialists to focus on interpreting the data, rather than collecting it. Data collected for each ICU patient includes the following: demographic characteristics on admission, reason for admission, visit history, medicationrelated allergies, and list of medications before admission, as documented in PharmaNet (a provincial database containing data about prescriptions dispensed in $\mathrm{BC}$ community pharmacies). The CPST ensures that a hard copy of the PharmNet documentation is included with the patient monitoring form and is inserted into the patient's medical record. The CPST also monitors information about intravascular devices, such as time of placement, location, and type of access (e.g., central or arterial lines), and availability of a urine catheter or nasogastric or orogastric tubes. In addition, medication information for each patient, such as drug, dose, route, frequency, and start and stop dates, is collected daily by the CPST and transferred to the standard monitoring form. This activity is critical because of the unit's current lack of a computer-generated medication administration record. Various predefined laboratory and microbiological parameters, such as electrolytes, hematology and liver profile, coagulation parameters, and culture and sensitivity results, are also updated by the CPST on the patient monitoring form. These data are collected at the start of the day, after patient care rounds, and on an as-needed basis. 


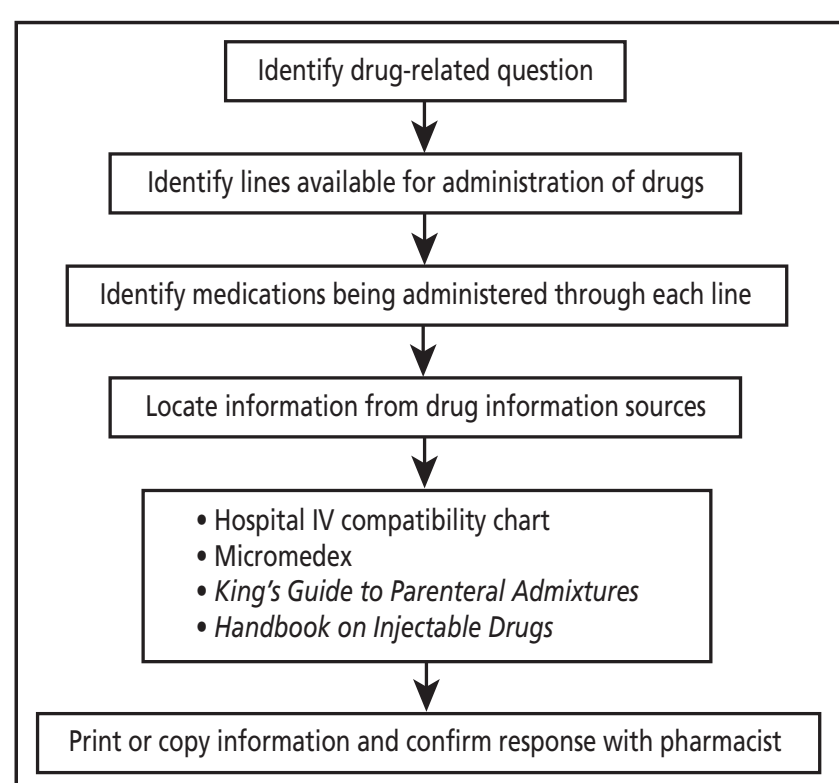

Figure 1. Compatibility protocol for IV drugs.

Any new abnormalities in laboratory parameters, especially those reported at a predefined critical value requiring action, or results of new microbiological cultures arising from regular checks throughout the day are brought to the attention of a clinical pharmacy specialist immediately and highlighted on the patient monitoring form. This allows the pharmacist to make drug-related decisions based on the most up-to-date information.

\section{Therapeutic Drug Monitoring}

The CPST is trained to assist in providing therapeutic drug monitoring services for medications such as vancomycin, aminoglycosides, phenytoin, digoxin, and any other medication for which monitoring is requested by the clinical pharmacy specialist. Patient-specific information about these drugs is collected by the CPST and transcribed to a standardized therapeutic drug monitoring form (see Online Appendix 2, at www.cjhp-online.ca/index.php/cjhp/issue/view/72/showToc). Therapeutic drug monitoring for a particular drug is performed daily for as long as the patient is receiving the medication. Other information collected includes trends from blood work, vital signs, medication administration times, serum drug concentrations, any documented seizure activity (for patients receiving an anticonvulsant), and any sources of cooling (such as antipyretics for patients who are taking antibiotics). This activity requires the CPST to review each patient's medical record and daily flow sheet and to interact with other health care professionals. The clinical pharmacy specialists are notified immediately when a serum drug concentration becomes available and in any instance of predefined critical values or missing doses, and the information is highlighted in the therapeutic drug monitoring form. The clinical pharmacy specialist benefits from this support activity because all pertinent information is up to date and readily available when a decision is needed regarding a patient's drug therapy. In addition, most information required for a detailed clinical pharmacy note can be obtained from the therapeutic drug monitoring and patient monitoring forms.

\section{Ward-based Troubleshooting}

Throughout the day, the CPST performs various troubleshooting functions in the ICU, activities that were previously handled by the clinical pharmacy specialist. These troubleshooting activities might include dealing with issues related to ward stock or narcotics, handling drug-distribution problems, expediting "stat" medication orders, clarifying physicians' orders, looking for missing medications, documenting use of Special Access Programme drugs and study drugs, investigating drug misadventures, and documenting unusual medication-related incidents. The CPST consults with the clinical pharmacy specialist as needed to solve these problems. By having the CPST serve as the initial pharmacy contact person for staff from the ICU and centralized pharmacy dispensary, issues can be quickly triaged, with the clinical pharmacy specialist becoming involved only for issues specifically requiring his or her input.

\section{Compatibility of Drugs for IV Administration}

A typical patient in the ICU might be receiving multiple drugs by IV administration throughout the day. These medications may be administered either on an intermittent dosing schedule or by continuous infusion. Nursing staff with questions about IV drug compatibility may approach the wardbased pharmacists for help. These questions can come at various times of the day, including during patient care rounds. The CPSTs at the Royal Columbian Hospital are trained to search for the answers to these IV compatibility questions using a stepwise approach (Figure 1). The CPST first determines the specific IV lines available and the medications being infused through each line. Next, the CPST searches for compatibility information in reference books, IV compatibility charts, or in electronic resources and prints a copy, which is made available for the pharmacist or nurse to add to the patient's medical record. The CPST then applies the information obtained to the medications that the patient is receiving and identifies the best option. All information is presented to the clinical pharmacy specialist for discussion and approval.

\section{Reporting of Adverse Drug Reactions}

Patients sometimes experience an adverse drug reaction during their hospital stay, and the pharmacist may choose to document and submit these cases to Health Canada. The CPSTs can assist the pharmacist by filling in some details on the adverse drug reaction reporting form, such as the patient's demographic characteristics, information about the health product, and contact information; sometimes the CPST is even able to document the entire case. The CPST gives the partly (or 
fully) completed form to the clinical pharmacy specialist; once the form has been completed and approved by the clinical pharmacy specialist, the CPST goes on to fax and file the report.

\section{Student Orientation}

CPSTs have taken over the role of student orientation during their first day of clinical rotation. In addition to their typical patient care activities, the pharmacists at the Royal Columbian Hospital are regularly asked to orient students as part of undergraduate, residency, and doctor of pharmacy programs. At the start of the rotation, typically on a Monday morning, the clinical pharmacy specialist would spend a few hours familiarizing the student to the unit and its staff, patient and drug information sources, the in-house computer program, and the patient monitoring form. Now that this task has been delegated to the CPST, the clinical pharmacy specialist's time on these days can be better spent preparing for patient care rounds.

\section{Support in Case of Reduced Pharmacist Coverage}

On occasion, only one clinical pharmacy specialist is available to cover both of the ICU care teams. Reasons for this situation include illness, attendance at meetings, teaching sessions at the Faculty of Pharmaceutical Sciences, or other situations when back-filling cannot be provided. The CPST can minimize the impact of reduced pharmacist coverage in the ICU by helping the remaining pharmacist to triage and deal with issues for the other ICU team. The CPST liaises with the covering clinical pharmacy specialist and discusses issues that might be expected to arise during the day, including pending results for serum drug concentrations, critical laboratory values, pharmacy consults, new admissions, transfers, and any clinical issues brought up by the pharmacy dispensary staff. The CPST updates the patient monitoring and therapeutic drug monitoring forms for all patients as usual, thus ensuring that the covering pharmacist has all the information needed for effective troubleshooting. All of these activities are performed in collaboration with the covering clinical pharmacy specialist.

\section{Projects}

Several large projects undertaken recently at the Royal Columbian Hospital have involved the CPST. The ICU recently moved to a new location, and the CPSTs were in charge of organizing the drug-distribution system in the new unit and transferring ward stock, narcotics, and patient-specific drugs during the move. Having the CPSTs take charge of the move allowed the clinical pharmacy specialists to continue focusing on their patient care duties.

Another project with which the CPSTs were involved at the time of writing was the roll-out of the computer-generated medication administration record (MAR) in the ICU. Various meetings were held to plan the logistics of this initiative and to discuss potential issues with the nursing staff, and in this regard the CPSTs have been the main liaison between the nursing staff and pharmacy department. In addition, the CPSTs have been responsible for many of the prerequisite background tasks related to the information system, such as entering preprogrammed order sets and evaluating the format of the MAR. Any issues related to generation and formatting of the MAR that have arisen because of the change in process are now reviewed and solved by the CPST liaison.

\section{BENEFITS OF THE CPST PROGRAM}

The primary benefit anticipated from any CPST program is an increase in the ability to provide comprehensive pharmaceutical care. A multifacility health care system in Pittsburgh evaluated its clinical technician program in terms of the number of patients assessed in a day and the number of interventions performed by a pharmacist toward targeted initiatives. ${ }^{1}$ After 2 years, the investigators observed a 3 -fold increase in the number of patients seen in a day (from 23 to more than 90 patients). ${ }^{1}$ In addition, the satisfaction of both pharmacists and pharmacy technicians increased, which was attributed to improvements in efficiency and expanded scope of clinical services. ${ }^{1}$

Incorporating a CPST program in the delivery of direct patient care in an adult tertiary-level ICU can improve pharmacists' work efficiency in a number of ways, as exemplified by the program described here. First, the clinical pharmacy specialists were able to increase the number of comprehensive patient assessments per day. Initially, each pharmacist had to assess 13 patients within the 2 -h period before patient care rounds. This was challenging, because an experienced pharmacist needs about $15 \mathrm{~min}$ to perform a standard comprehensive assessment for each patient. As such, each clinical pharmacy specialist typically completed a full assessment for only 8 patients before patient care rounds. After initiation of the CPST program, the time spent for data collection by the pharmacist was reduced by about 5 min per patient, and the clinical pharmacy specialists increased to 12 the number of comprehensive assessments they could perform in the time available.

Second, the clinical pharmacy specialist could spend more time performing cognitive-based activities throughout the day, with minimal time used for data collection. The CPST assisted the pharmacists in technical functions such as data collection, patient triage, ward-based troubleshooting, student orientation, and various projects. The main impact has been evident in the morning, when there is typically limited time to assess patients before multidisciplinary patient care rounds. There have also been fewer interruptions during patient care rounds, since the CPST can solve drug-related issues that do not require input from a pharmacist.

Third, assistance from the CPST for data collection in the morning allows the clinical pharmacy specialists to start their shifts later. Before initiation of the CPST program, the clinical pharmacy specialists arrived at 0700 in the morning to perform detailed patient assessments, including collection of data. If patient care rounds extended late into the afternoon, the pharmacists would have to stay later. It is valuable to have the clinical pharmacy specialist stay for all patient care rounds, to 
allow them to provide prospective input into decisions about patient care and to ensure that appropriate decisions are made about drug therapy, but this meant extended work hours for the pharmacists. Once the CPST took over data collection activities in the morning, the pharmacists were able to start later, and their work hours became more compatible with situations in which rounds extend late into the afternoon. Furthermore, the CPST can free up time for the clinical pharmacy specialist in the afternoon to perform other patient care-related activities. Overall, this leads to a reduction in overtime worked by the clinical pharmacy specialist.

Finally, when clinical pharmacy coverage in the ICU is reduced, the CPST helps the remaining pharmacist to manage the workload. Information for all ICU patients is up to date, and less time is required to collect data for patients under the care of the other multidisciplinary team.

All of these benefits became apparent through a comparison of the periods before and after initiation of the CPST program. We continue to monitor the impact of the program as it evolves.

\section{CONSIDERATIONS IN IMPLEMENTING A CPST PROGRAM}

A CPST program can be implemented in almost any practice setting where a pharmacist performs patient care activities. Each institution must determine the best utilization of its resources and should consider a number of factors when implementing such a program.

First, there is limited information to date on the activities that CPSTs have successfully performed. The available literature on the performance of nontraditional activities by pharmacy technicians is scant and does not provide detailed descriptions. ${ }^{1-5}$ Therefore, institutions wishing to initiate a CPST program must be prepared to conduct research about their own staff and their daily workloads to determine the best use of the technicians. Second, most of the CPST duties will be assigned according to the overall capabilities and skills of the candidates. The candidate must be familiar with the institution's drugdistribution processes and must be enthusiastic about taking on new roles. Because the duties involved are new responsibilities for technicians, a formal training program may be required. Third, maintaining consistency of services is important. An adequate number of CPSTs must be available for coverage of services, including sick calls and holidays. In addition, training and procedures should be consistent, to ensure that the CPSTs can perform their duties consistently, thus allowing for seamless rotation of pharmacists and technicians. Efficiencies would be lost if a transition period were required with staff changes at the beginning of every week. Finally, formalized procedures and protocols are necessary to ensure standardization of the services delivered by CPSTs. These procedures and protocols must be updated and disseminated regularly according to the tasks, activities, and challenges identified in the program.
When a CPST program is implemented in a department where technicians represent the majority of the pharmacy team, it is important to promote the new roles as an opportunity for professional growth for experienced members. During this era of developments in technician training and certification standards, the perception of a tiered technician system may generate animosity among staff performing strictly distribution-related duties. A focus on the CPST position as an opportunity for career advancement is one way of maintaining positive team dynamics in the pharmacy department.

\section{CONCLUSIONS}

Pharmacy technicians with additional training and support in the clinical area increase the work efficiency of pharmacists in the ICU, thereby making it possible to extend pharmacy services in direct patient care to a larger number of patients during the pharmacists' work day.

\section{References}

1. Ervin KC, Skledar S, Hess MM, Ryan M. Data analyst technician: an innovative role for the pharmacy technician. Am J Health Syst Pharm 2001;58(19):1815-1818.

2. Keresztes JM. Role of pharmacy technicians in the development of clinical pharmacy. Ann Pharmacother 2006;40(11):2015-2019.

3. Weber E, Hepfinger C, Koontz R, Cohn-Oswald L. Pharmacy technicians supporting clinical functions. Am J Health Syst Pharm 2005;62(23): 2466-2472.

4. Academy of Managed Care Pharmacy. White paper on pharmacy technicians 2002: needed changes can no longer wait. Am J Health Syst Pharm 2003;60(1):37-51.

5. Ambrose PJ, Saya FG, Lovett LT, Tan S, Adams DW, Shane R. Evaluating the accuracy of technicians and pharmacists in checking unit dose medication cassettes. Am J Health Syst Pharm 2002;59(12):1183-1188.

Vincent H Mabasa, BSc(Pharm), ACPR, PharmD, is a Clinical Pharmacy Practice Leader at the Burnaby Hospital, Burnaby, British Columbia.

Douglas L Malyuk, BSc(Pharm), ACPR, PharmD, is a Clinical Pharmacy Specialist - Intensive Care, at the Royal Columbian Hospital, New Westminster, British Columbia.

Anthony Tung, $\mathrm{BSC}($ Pharm), ACPR, is a Clinical Pharmacist with the Surrey Memorial Hospital, Surrey, British Columbia.

Robert M Balen, BSc(Pharm), ACPR, PharmD, is the Manager of Pharmacy Services at the Royal Columbian Hospital, New Westminster, British Columbia.

Tricia R Nicolls is a Clinical Pharmacy Support Technician with the Royal Columbian Hospital, New Westminster, British Columbia.

Nicole L Rahiman is a Clinical Pharmacy Support Technician with the Royal Columbian Hospital, New Westminster, British Columbia.

\section{Address correspondence to:}

Dr Vincent H Mabasa

Pharmacy

Burnaby Hospital

3935 Kincaid Street

Burnaby BC V5G 2X6

e-mail: Vincent.Mabasa@fraserhealth.ca

\section{Acknowledgements}

We acknowledge the following people for their constant support of the clinical pharmacy support technician program: Dr Shallen Letwin, Bruce Millin, Dr Wendy Gordon, Dr Sean Keenan, Karen Watson, and the rest of the ICU/CCU and pharmacy staff at the Royal Columbian Hospital. 